\title{
A Dedicated Backscattered Electron Detector for High Speed Imaging and Defect Inspection
}

Maximilian Schmid ${ }^{1, *}$, Andreas Liebel ${ }^{1}$, Robert Lackner $^{1}$, Daniel Steigenhöfer ${ }^{1}$, Adrian Niculae ${ }^{1}$, Heike Soltau $^{1}$

1. PNDetector GmbH, München, Germany

Pattern analysis and defect detection is an important field of quality control in modern semiconductor production. High device densities, small structures and increasingly complicated process operations demand reliable and fast wafer inspection. At the same time the influence on the specimen should be kept to a minimum to ensure damage-free process control. Defect and pattern analysis algorithms are capable of fulfilling this demand. However, the capabilities of these software tools depend on fast detection systems providing high speed and high quality images. Here, we show a Backscattered-Electron (BSE) detector dedicated to imaging with signal rise times down to $10 \mathrm{~ns}$ in superior quality. This allows ultra fast detection resulting in minimal exposure time of the specimen.

To support the high speeds of the BSE-Chip, the diode and the preamplifier board form one unit placed in a flat high-grade housing. Figure 1 shows the BSE detector module. The device is compatible with high vacuum conditions inside Scanning Electron Microscopes (SEM) and the materials are selected carefully to not influence the electron beam. With it's thickness of only $2.5 \mathrm{~mm}$, it can be placed directly under the pole piece of an SEM. At this position it offers small working distances (WD) with high Geometric Collection Efficiencys (GCE) for the BSE. For example, equipped with a BSE diode with $1 \mathrm{~mm}$ inner hole and 40 $\mathrm{mm}^{2}$ sensitive area the module exhibits a maximum GCE of $60 \%$ at a distance of $2 \mathrm{~mm}$ between sample and Diode. Such high GCE enable high signal outputs even at low beam currents compared to other BSE detectors.

The close connection of the BSE-Diode to the preamplifier board ensures minimum stray capacities between the signal generation and amplification. In addition, the design of the amplifier is geared to the diode, which enables the BSE detector to realize rise times down to $10 \mathrm{~ns}$, at an amplification factor of $10^{4}$ V/A. Figure 2 shows the step response of the BSE detector module. The edge was simulated by an ultra fast switching LED with switch on times around $7 \mathrm{~ns}$. Corresponding to this input our BSE detector module shows a rise time of about $10 \mathrm{~ns}$ to $15 \mathrm{~ns}$. As an example, an image of 1000x1000 pixels can be taken within $10 \mathrm{~ms}$, highlighting the outstanding speed of the BSE detector module and the minimal interaction time between the electron beam and the specimen.

Figure 3 shows two SEM images of a defect recorded with the BSE detector module at 100 ns dwell time at $4 \mathrm{keV}$ beam energy and $120 \mathrm{pA}$ beam current. The advantage of BSE over Secondary Electron (SE) detection in defect analysis becomes visible since, next to contaminations with foreign particles, the topographic structure of the specimen can be monitored. Figure 3(a) is a compositional image with pure material contrast and figure 3(b) the corresponding topographical image. Since the BSE detector module provides each signal of the four segments of the diode separately, different signal combinations can be monitored in a simultaneous scan. This advantage further decreases the exposure time of the specimen to the electron beam. 


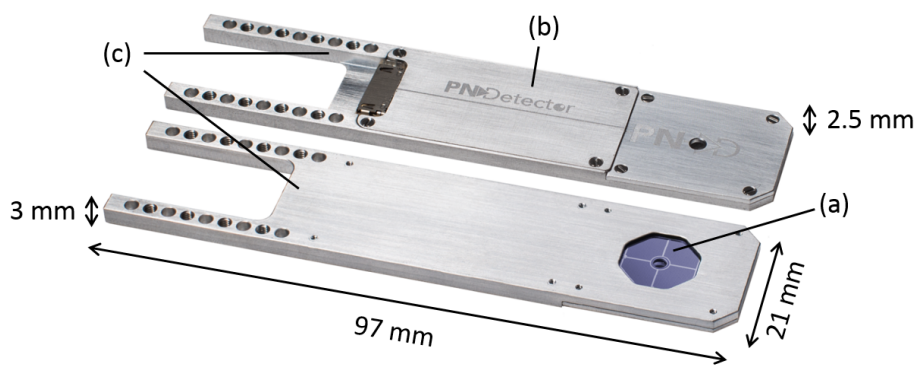

Figure 1. Front and backside view of the PN-Detector high speed BSE detector module: (a) 4 Quadrant BSE Diode, (b) 4 channel preamplifier, (c) ultra flat aluminum housing.

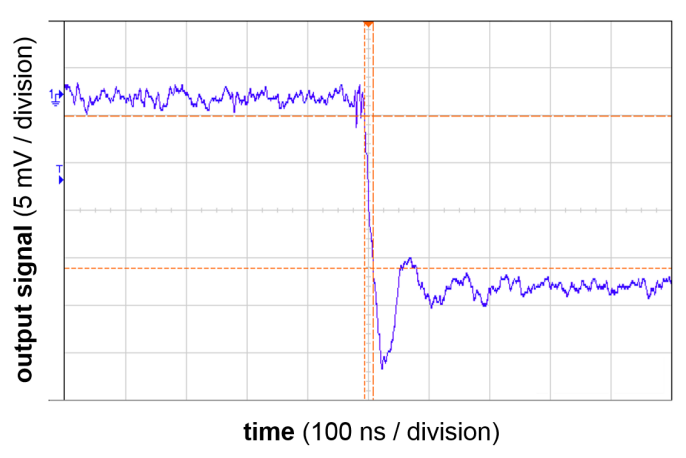

Figure 2. BSE detector module output vs. time. Signal rise time of 10 to $15 \mathrm{nsec}$ measured by illuminating the BSE-detector with a high speed LED pulser with a switch on rise time of $7 \mathrm{~ns}$.
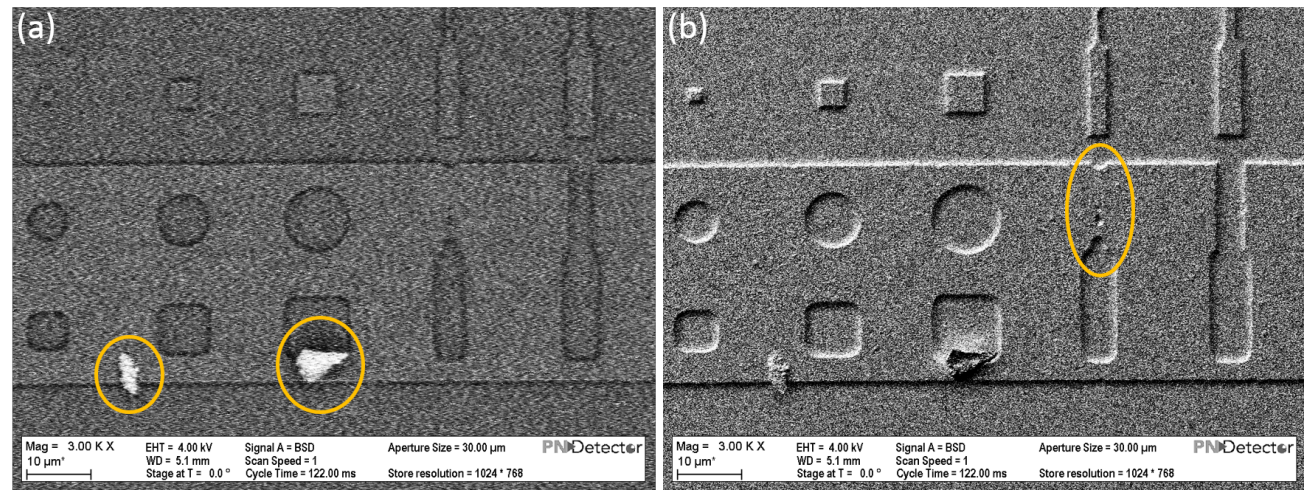

Figure 3. SEM images of a structured $\mathrm{Al}$ layer on a $\mathrm{Si}$ wafer. Image taken at a beam energy of $4 \mathrm{keV}$ with a beam current of $120 \mathrm{pA}$ at a working distance of $5.1 \mathrm{~mm}$ and a pixel dwell time of $100 \mathrm{~ns}$. (a) Compositional contrast, high Z particle contamination highlighted. (b) Topographical contrast, structural defect highlighted. 